\title{
Estudio paramétrico de la respuesta termo-estructural de un puente metálico multijácena sometido a incendios reales
}

\section{A parametric study on the thermo-mechanical response of a multi-girder steel bridge submitted to real fires}

G. Peris-Sayol $^{(*)}$, J. Alós-Moya ${ }^{(*)}, \underline{\text { I. Payá-Zaforteza }}^{(*)}$, A. Hospitaler-Pérez ${ }^{(*)}$

\section{RESUMEN}

La respuesta de los puentes frente al fuego es un tema poco estudiado y ausente de las normativas actuales a pesar de la frecuencia y consecuencias de los incendios en puentes. Por ello, este artículo analiza la respuesta de un puente metálico multijácena ante incendios reales provocados por un camión cisterna. Para ello se realizan primeramente modelos de incendio del puente y su entorno con técnicas de Dinámica de Fluidos Computacional y luego análisis termo-estructurales no lineales de las vigas más afectadas por el fuego. El estudio analiza aspectos de modelización numérica y la influencia de la posición del camión y la existencia de derrames de combustible. Los resultados son de interés para ingenieros e investigadores y muestran (a) la importancia de considerar condiciones reales de incendio y no modelos simplificados y (b) la necesidad de desarrollar herramientas específicas para el proyecto de puentes frente al fuego.

Palabras clave: Fuego; puente; Dinámica de Fluidos Computacional; CFD; diseño basado en prestaciones.

\section{ABSTRACT}

Bridge fire response is a barely studied topic not covered in current design codes despite the frequency and consequences of this kind of events. Within this context, this paper analyzes the response of a multi-girder steel bridge in real fires caused by a tanker. To reach this goal, fire models of the bridge and its surroundings are done using Computer Fluid Dynamics (CFD) techniques, and then an uncoupled thermo-mechanical analysis of the most fire affected bridge girders is carried out. The study analyzes modeling features as well as the influence of the position of the tanker and the existence of gas spills. Results are interesting for both researchers and engineers and show (a) the importance of considering real fires and not simplified models, and (b) the need to develop a performance based approach specific to bridge fire design.

Keywords: Fire; bridge; Computer Fluid Dynamics; CFD; performance based approach.

(*) ICITECH - Universitat Politécnica de València, (España).

Persona de contacto/Corresponding author: igpaza@upv.es (I. Payá-Zaforteza)

Cómo citar este artículo/Citation: Peris-Sayol, G., Alós-Moya, J., Payá-Zaforteza, I., Hospitaler-Pérez, A. (2014). Estudio paramétrico de la respuesta termo-estructural de un puente metálico multijácena sometido a incendios reales. Informes de la Construcción, 66(EXTRA-1): mo02, doi: http://dx.doi.org/10.3989/ic.13.077.

Licencia / License: Salvo indicación contraria, todos los contenidos de la edición electrónica de Informes de la Construcción se distribuyen bajo una licencia de uso y distribución Creative Commons Reconocimiento no Comercial 3.o. España (cc-by-nc). 


\section{INTRODUCCIÓN}

Los puentes son elementos críticos de las infraestructuras de transporte cuyo colapso o deterioro puede tener grandes repercusiones económicas y sociales. Para minimizar la probabilidad de que estos eventos puedan suceder, las diferentes normativas -véase por ej. (1) (2)- definen las acciones o cargas a tener en cuenta en su diseño. En este contexto, acciones extraordinarias como el sismo, el viento o la socavación de la cimentación han sido muy investigadas en el pasado -véase por ej. (3)- y están ampliamente recogidas en las normativas actuales. En cambio, estas normativas no indican nada sobre cómo proteger o diseñar un puente frente al fuego. Así, normas relativas al diseño frente al fuego como la parte 1-2 del Eurocódigo 1 (4) describen las acciones térmicas y mecánicas para el proyecto de edificios y por lo tanto excluyen los puentes. Por su parte, las normas de acciones en puentes como el Eurocódigo 1 parte 2 (2) especifican aspectos como las sobrecargas de uso, pero no indican cómo considerar el fuego en el proyecto o evaluación estructural de un puente. Análogamente, la comunidad científica ha prestado mucha más atención a los efectos del fuego en los edificios -véase por ej. (5) (6) (7) (8)- que a los efectos del fuego en los puentes como demuestran Garlock et al. (9).

Este vacío contrasta con la importancia del fuego en los puentes evaluada por el número y consecuencias de los incidentes ocurridos en el pasado. Así, una encuesta realizada por el Departamento de Transportes del Estado de Nueva York de los Estados Unidos (10) reveló que el fuego fue la quinta causa de colapsos de puentes en este país, por delante de eventos como el sismo. Las consecuencias de un incendio en un puente son ilustradas por el colapso del Intercambiador Mc Arthur en Oakland, California, tras el incendio causado por el vuelco de un camión cisterna el 29 de abril del año 2007 (Figura 1). La reparación del puente costó 9 millones de dólares, pero más importantes aun fueron los costes generados en el área de la Bahía de San Francisco por el cierre del intercambiador que se estimaron en 6 millones de dólares por día de cierre. Otro ejemplo ilustrativo es el del incendio de un puente en las proximidades de Robbinsville (Nueva Jersey, USA) el 3 de octubre de 2012. En este caso el puente no colapsó, pero el accidente produjo el cierre de más de $73 \mathrm{~km}$ de una de las autopistas más importantes de los Estados Unidos, alteraciones de tráfico en tres estados norteamericanos e interrupciones del tráfico durante seis semanas (11). Una completa revisión bibliográfica sobre los efectos del fuego en puentes, incidentes ocurridos y estudios previos en el tema puede encontrarse en Garlock et al. (9) y Payá-Zaforteza et al. (12).

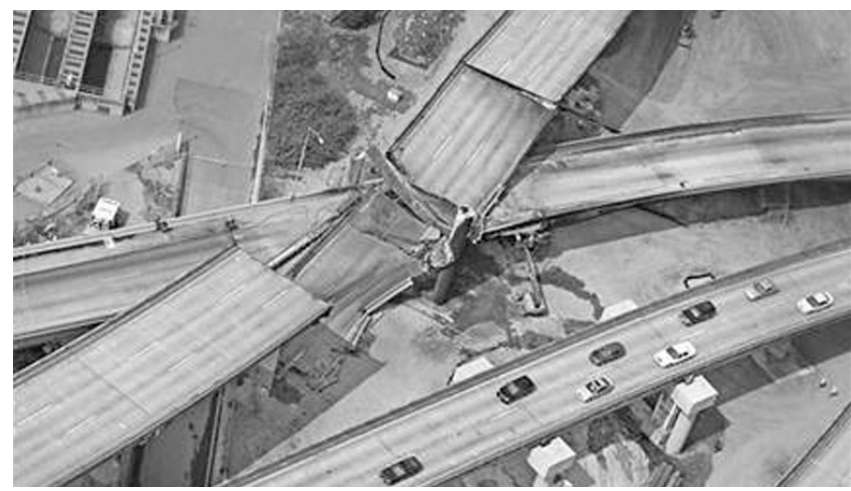

Figura 1. Imagen aérea del estado en que quedó el Intercambiador Mc Arthur en Oakland tras el incendio sufrido el 29 de abril del año 2007. Fotografía de Robert Campbell usada con su permiso.
En este artículo se emplean modelos numéricos para estudiar la respuesta de un puente metálico ante diferentes escenarios reales de incendios causados por camiones cisterna que transportan combustibles. El puente analizado se describe en el apartado 2 del artículo, mientras que en el apartado 3 se presentan las diferentes posiciones del camión cisterna que definen potenciales escenarios de fuego y las técnicas de Dinámica de Fluidos Computacional (Computer Fluid Dynamics, CFDs) empleadas para simular el incendio y obtener las temperaturas en el gas alrededor del puente. Estas temperaturas son el punto de partida de un análisis termo-estructural desacoplado presentado en el apartado 4 que considera las no linealidades geométricas y del material.

Los resultados de este análisis se presentan en el apartado 4.6 y son comparados con un modelo de calentamiento estandarizado (curva de hidrocarburos aplicada de forma uniforme en toda la longitud del puente) empleado por Payá-Zaforteza y Garlock (13) que no se corresponde con una situación real de incendio. La comparación de resultados permite analizar la precisión y seguridad obtenidas al emplear la curva de hidrocarburos (un fuego simplificado) en lugar de modelos de cálculo basados en el uso de CFDs. Además, el estudio analiza la influencia de diferentes parámetros como la discretización de la carga de fuego, la posición del camión que causa el incendio y la existencia de derrames de combustible. Por todo ello, el trabajo que se presenta aquí tiene un gran interés investigador (por la novedad del tema tratado) y práctico (por la aplicabilidad de los resultados obtenidos).

\section{DESCRIPCIÓN DEL PUENTE ANALIZADO}

El puente analizado en este estudio es un puente biapoyado proyectado por la Federal Highway Administration (FHWA) de los Estados Unidos de América. El puente salva una luz de 12,2 m y su planta y sección transversal se muestra en la Figura 2. La sección transversal está formada por cinco vigas de acero del tipo W33x141 según el catálogo de perfiles del American Institute of Steel Construction (AISC) (14) que soportan una losa de hormigón armado de 0,2 m de canto y 12,97 metros de anchura. La losa no está conectada a las vigas, por lo que no se trata de una estructura mixta. Además, existen diafragmas en las secciones de centro luz y de apoyos que proporcionan estabilidad lateral al puente. El tablero tiene dos juntas de dilatación en sus extremos con un ancho de 3,6 cm. La separación entre estribos es de 11,8 m y el gálibo del puente es de 5,1 m. El acero empleado es del tipo A36 de acuerdo con (15) y, por tanto, su límite elástico garantizado a temperatura ambiente es de $250 \mathrm{MPa}$.

La respuesta de esta estructura ante diferentes escenarios de incendio se analiza con modelos numéricos en los apartados 3 y 4 del artículo. El análisis sigue las tres fases siguientes:

(a) Simulación de incendios mediante el software Fire Dynamics Simulator (FDS) (16) en el que se obtienen las temperaturas producidas por el incendio en el entorno del puente.

(b) Obtención de las temperaturas en la superficie y en el interior de la viga del puente más desfavorable a partir de las temperaturas existentes en su perímetro. Para ello se resuelve un problema de transferencia de calor con el programa Abaqus (17).

(c) Obtención de la respuesta estructural de la viga más solicitada también mediante Abaqus (17). En esta fase, se supone 


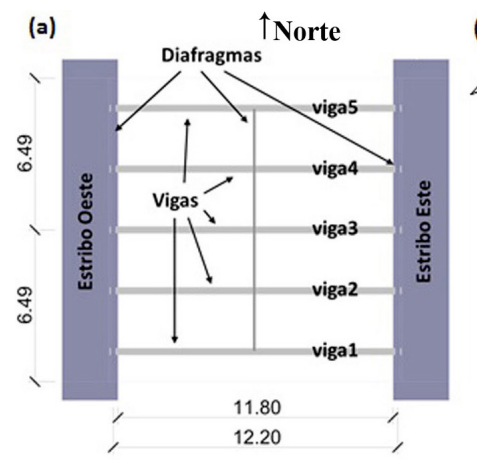

(b)

Figura 2. (a) Planta y (b) sección transversal del puente estudiado.

la viga sometida a cargas gravitatorias constantes y a un incremento de temperaturas que implican un deterioro progresivo de las características de los materiales.

Se insiste en que en la fase (a) se trabaja con un modelo de todo el puente y de su entorno, mientras que en las fases (b) y (c) se trabaja solamente con la viga de la estructura más solicitada.

\section{MODELO DE FUEGO CON TÉCNICAS DE DINÁMICA DE FLUIDOS COMPUTACIONAL}

El modelo de FDS (16) resuelve numéricamente, en un volumen de control en el que se ha definido un mallado, una forma de las ecuaciones de Navier-Stokes apropiadas para baja velocidad del flujo térmico, con un especial énfasis en el humo y el transporte de calor por el fuego. FDS calcula la temperatura, densidad, presión, velocidad, flujo de calor, tasa de pérdida de masa y composición química en cada celda de la malla en cada paso de tiempo discreto. Para ello el programa emplea (a) la ecuación de la conservación de la masa, (b) la ecuación de la conservación de las especies, (c) la ecuación de la conservación de la cantidad de movimiento, (d) la ecuación de la conservación de la energía y (e) la ecuación de estado. Adicionalmente se ha recurrido al modelo de turbulencia LES (Large Eddy Simulator) asumiendo la forma de Smagorinsky.

A partir del análisis de incidentes previos -véase Garlock et al. (9) y Alós-Moya et al. (18)-, se han planteado seis escenarios de fuego (Figura 3) en los que se han considerado las distribuciones de carga de fuego más importantes en el ámbito de los puentes y que se refieren al incendio de un camión bajo el puente con o sin derrame de combustible. Atendiendo a la distribución de la carga de fuego, se diferencian tres posibilidades básicas: camión cisterna en centro luz - casos (a) y (d) en la Figura $3^{-}$, camión cisterna en lateral de vía -casos (b) y (e) en la Figura 3- y camión cisterna en centro luz con derrame de combustible lateral - casos (c) y (f) en la Figura 3-. Este patrón se ha seguido para dos posiciones: (i) huella de la carga de fuego contenida bajo el puente y (ii) huella de la carga de fuego parcialmente situada bajo el puente. El camión en llamas se ha modelizado de forma simplificada por una superficie horizontal de $30 \mathrm{~m}^{2}(12 \times 2,5 \mathrm{~m})(19)$ dispuesta a un metro de altura con una tasa de liberación de calor (HRR) de $2400 \mathrm{KW} / \mathrm{m}^{2}$, correspondiente a una piscina de gasolina con (a) fire1

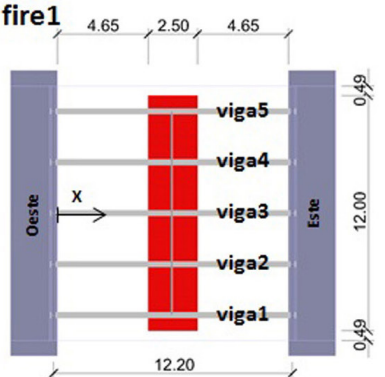

(d) fire4

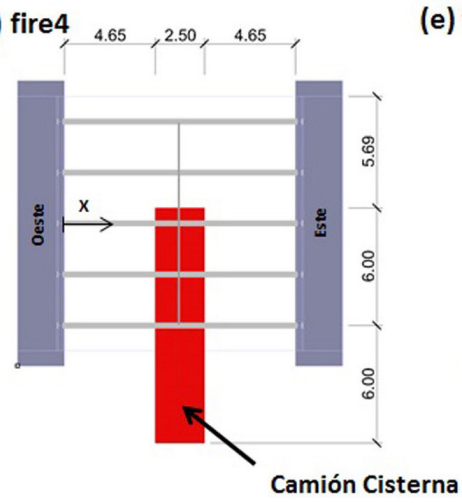

(b) fire2

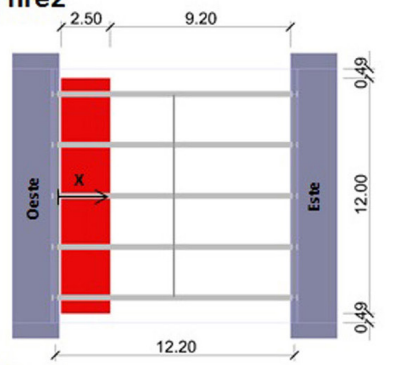

(e) fire5

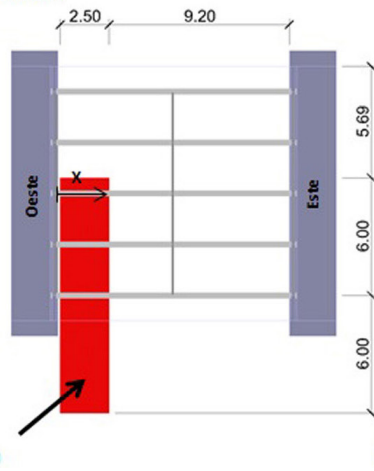

(c) fire3

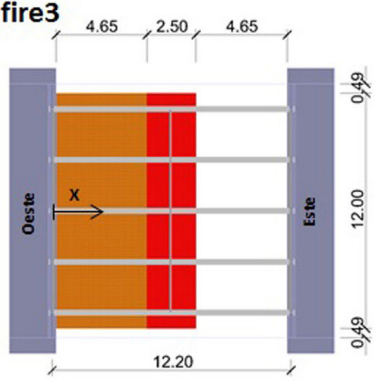

(f) fire6

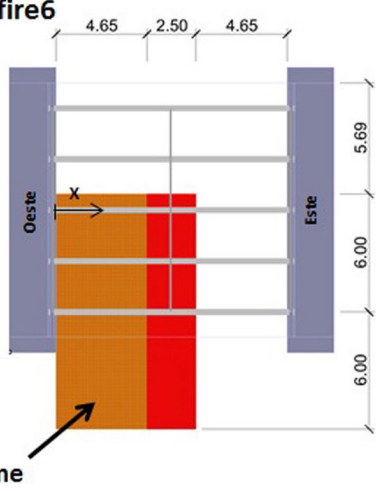

Figura 3. Posiciones de las cargas de fuego: (a) fire1: centro luz - viga central, (b) fire2: próxima estribo - viga central, (c) fire3: centro luz con derrame - viga central, (d) fire 4: centro luz - viga extrema,

(e) fire5: Próxima estribo- viga extrema, (f) fire6: centro luz con derrame - viga extrema. 
(a)

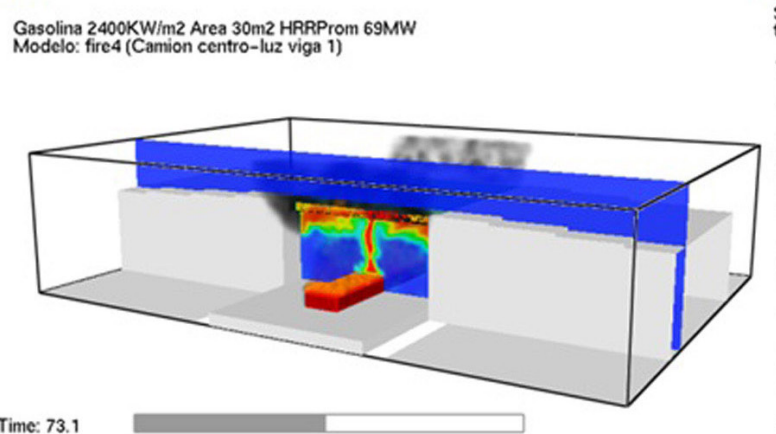

(b)

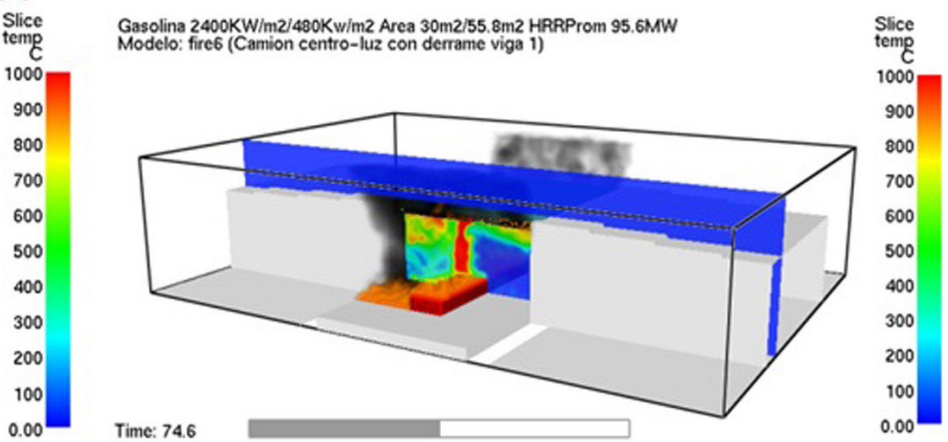

Figura 4. Modelos FDS para dos casos con la carga centrada en la viga 1: (a) fire4 y (b) fire6.

diámetro equivalente superior a 3 metros (20). El derrame lateral, de $55,8 \mathrm{~m}^{2}(12 \times 4,65 \mathrm{~m})$, se ha caracterizado mediante una superficie horizontal dispuesta a cota de rasante con una tasa de liberación de calor (HRR) de $480 \mathrm{KW} / \mathrm{m}^{2}$, correspondiente a una piscina de gasolina no confinada de pocos milímetros de espesor (20). En la Figura 4 se muestran las temperaturas registradas en un plano vertical centrado sobre la carga de fuego para los escenarios de fuego fire4 y fire6.

En la Figura 5 se muestran las temperaturas del gas en el entorno de la viga más solicitada térmicamente para los seis escenarios de fuego. Debido a las grandes diferencias de temperaturas según las zonas de exposición se decidieron obtener tres perfiles de temperaturas de acuerdo a las tres regiones de exposición: (a) Ala inferior, (b) Alma Sur y (c) Alma Norte.

\section{ANÁLISIS TERMO-MECÁNICO MEDIANTE ELEMENTOS FINITOS (FE)}

El estudio numérico se ha llevado a cabo mediante el software de elementos finitos (EF) Abaqus (17) y comprende el estudio de la respuesta de la viga más afectada en cada caso. Debido a la existencia de cargas de fuego no simétricas, ha sido necesario modelar la totalidad de la viga y no solo una mitad como se hizo en Paya-Zaforteza y Garlock (13). El análisis mediante Abaqus se ha abordado desacoplando el problema de transmisión de calor del problema estructural. Así, en una primera fase se obtienen las temperaturas en el entorno de la viga de estudio y en una segunda fase se analiza la respuesta estructural de la misma mediante la actualización de las características mecánicas de los materiales en función de su temperatura a lo largo del tiempo.

Para el análisis térmico se ha recurrido a un elemento finito de transferencia de calor tridimensional de ocho nodos con un grado de libertad por nodo (DC3D8) mientras que para el análisis estructural se ha utilizado un elemento finito tridimensional de ocho nodos con tres grados de libertad por nodo (C3D8). La utilización de elementos finitos tridimensionales viene motivada por la necesidad de capturar fenómenos locales como la abolladura del alma. Los coeficientes de convección y radiación utilizados son los propuestos por el Eurocódigo 1 (4). Para los modelos de fuegos reales se ha utilizado un coeficiente de $35 \mathrm{~W} / \mathrm{m}^{2}{ }^{\circ} \mathrm{K}$ mientras que para el caso de la curva de hidrocarburos se ha utilizado un valor de $50 \mathrm{~W} / \mathrm{m}^{2}{ }^{\circ} \mathrm{K}$. En ambos casos el valor de emisividad utilizado es 0,7.

La losa de hormigón únicamente se ha considerado en la transmisión de calor dada su influencia en el enfriamiento del ala superior de la sección metálica dejándose fuera del cálculo en el análisis estructural donde su contribución es despreciable por no existir acción mixta en el puente. (a) fire1 - viga 3

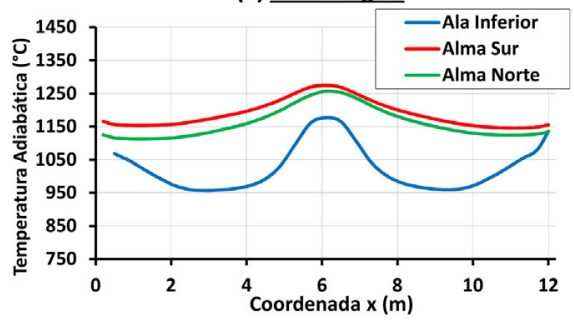

(d) fire4 - viga 2

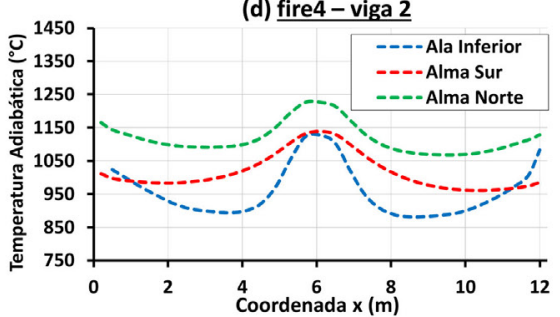

(b) fire2 - viga 3

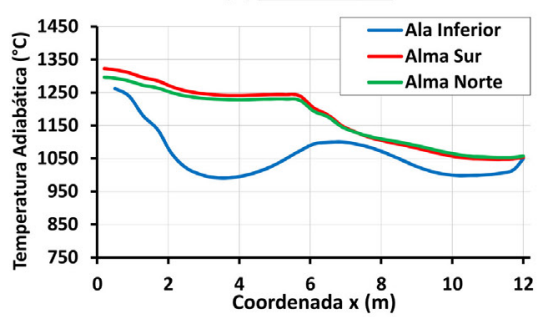

(e) fire 5 - viga 2

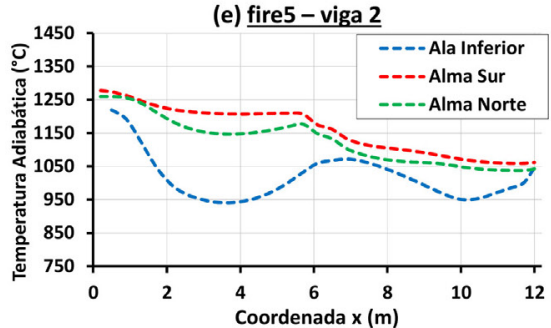

(c) fire3 - viga 3

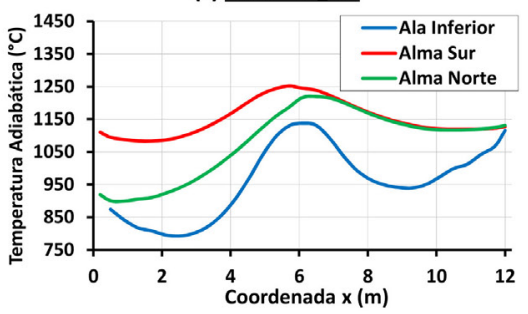

(f) fire6 - viga 2

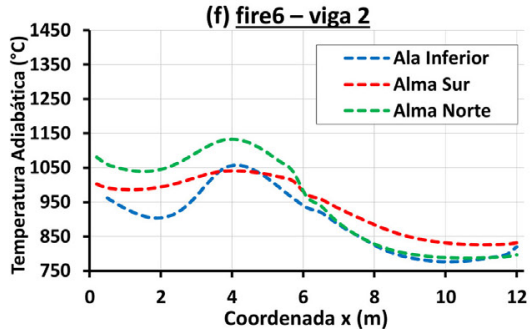

Figura 5. Temperaturas obtenidas mediante FDS en la viga más desfavorable para cada escenario.

En el eje de abcisas se representa la coordenada longitudinal x de la viga analizada (véase también la Figura 3 ). 


\subsection{Propiedades de los materiales}

Los materiales del puente, hormigón y acero, se han caracterizado con propiedades térmicas y mecánicas dependientes de la temperatura según lo indicado en los Eurocódigos 2 y 3 (21) (22). El acero utilizado es un A36 con límite elástico $250 \mathrm{MPa}$ en el que se ha incorporado el endurecimiento por deformación propuesto por el Eurocódigo 3 (22) y se han manejado curvas constitutivas reales $\left(\sigma_{n}, \varepsilon_{n}\right)$ obtenidas mediante las siguientes expresiones:

$$
\begin{gathered}
\sigma_{n}=\sigma \cdot(1+\varepsilon) \\
\varepsilon_{n}=\ln (1+\varepsilon)
\end{gathered}
$$

En lo que respecta al hormigón únicamente se han caracterizado las propiedades térmicas necesarias para el modelo de transmisión de calor (densidad, calor específico y conductividad) asumiendo para la conductividad el límite superior propuesto por el Eurocódigo 2 (21).

\subsection{Condiciones de contorno}

El puente estudiado tiene un único vano simplemente apoyado en sus extremos este y oeste. El apoyo este es fijo mientras que el oeste es móvil. Las restricciones a los movimientos en los apoyos fijo y móvil del modelo estructural no se aplican en un único punto o en una línea sino en una superficie. Esta superficie se ubica en la cara inferior del ala inferior, en la zona de contacto rigidizador-viga y tiene una de longitud $16 \mathrm{~mm}$ (espesor del rigidizador) y un ancho de $293 \mathrm{~mm}$ (ancho de la viga). Una explicación detallada de la modelización de los apoyos puede consultarse en PerisSayol et al. (23).

Además, es necesario tener en cuenta que la viga no puede dilatarse libremente de forma indefinida: cuando la magnitud del movimiento longitudinal del puente supera el ancho de la junta de dilatación, el movimiento es impedido por la existencia de un vano adyacente o de un estribo. Por ello, es necesario estudiar dos modelos estructurales diferentes en cada caso de carga: uno en el que no existe coacción al movimiento longitudinal (casos free con dilatación libre) y otro que incluya una restricción a la dilatación del tablero (casos fix). En este último caso, la viga se dilata libremente hasta que su desplazamiento longitudinal iguala el ancho de la junta de dilatación (36 mm en el extremo oeste).

\subsection{Análisis térmico}

Las cargas térmicas son las temperaturas del gas en el contorno de la viga a estudiar (la viga 3 en los escenarios fire1, fire2 y fire3 y la viga 2 en los escenarios fire4, fire5 y fire6). El empleo de temperaturas en los gases como acción térmica ha sido posible gracias al empleo de la superficie adiabática propuesta por Wickström (24). Este recurso no solo permite utilizar una única variable para incorporar efecto del calentamiento sino que además ha permitido desacoplar los modelos de fuego y termo-mecánico.

En el modelo estructural se han implementado siete cargas térmicas, seis de ellas procedentes de los modelos FDS (Figura 5) y una séptima es la curva de hidrocarburos indicada en el Eurocódigo 1 (4). Mientras que la curva de hidrocarburos se aplica de forma uniforme en todo el contorno, las cargas térmicas procedentes de FDS se han discretizado en tramos dadas las grandes variaciones que experimentan en el entorno de la viga. Por ejemplo, las temperaturas en el entorno del ala inferior de la viga 3 en el caso fire 3 oscilan entre los 790 y los $1140^{\circ} \mathrm{C}$, lo que hace poco realista aplicar una temperatura uniforme en toda su longitud. Un estudio paramétrico del número de tramos en los que debe discretizarse la viga se proporciona en el apartado 4.6.1.

El análisis térmico realizado en Abaqus proporciona las temperaturas en la superficie e interior de la viga analizada a lo largo del tiempo. A modo de ejemplo, la Figura 6a muestra la distribución de temperaturas en un corte vertical YY' de la viga 3 en la sección de centro luz para el caso fire1. En esta figura se aprecia como las temperaturas máximas en la sección se localizan en la región media del alma y como éstas resultan notablemente superiores a las que se registran en las alas. En la Figura 6b se aprecia que para un tiempo dado: (a) la temperatura en el centro del alma es superior a la de las alas y (b) el ala superior siempre presenta temperaturas inferiores al ala inferior como consecuencia no solo de estar sometida a menores temperaturas sino también a la gran inercia térmica del hormigón de la losa.

\subsection{Cargas gravitatorias}

Este estudio considera que no hay vehículos cruzando el puente mientras se produce el incendio. Esta hipótesis se basa en estudios anteriores -Paya-Zaforteza y Garlock (13) y Peris-Sayol et al. (23) - que han demostrado la poca influencia que tiene el nivel de sobrecarga en los tiempos y formas de colapso. Por tanto, las cargas gravitatorias consideradas en el presente estudio son:

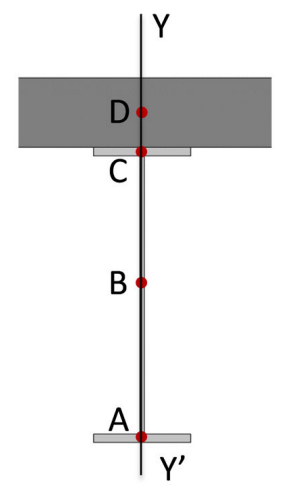

(a)

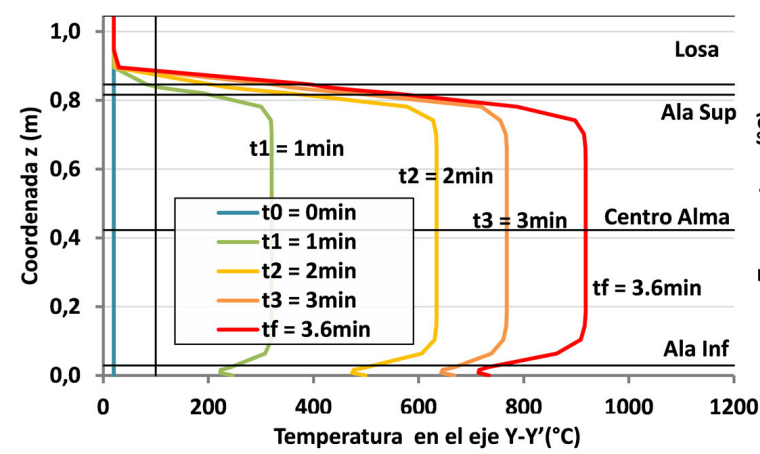

(b)

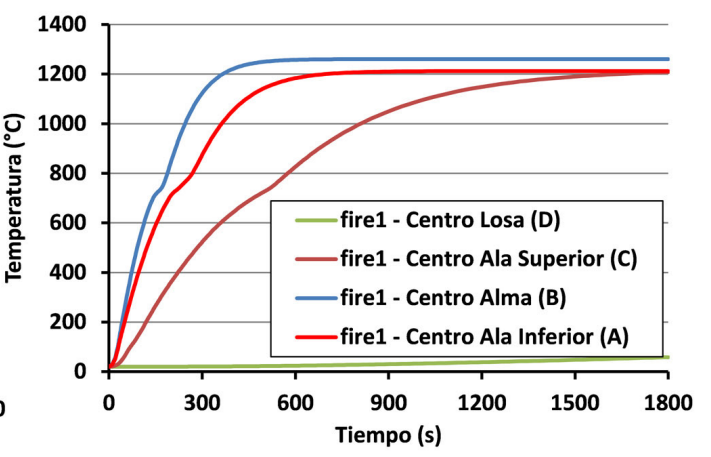

Figura 6. Temperaturas obtenidas mediante Abaqus en la viga 3 en el caso fire1: (a) en la sección de centro luz en distintos instantes temporales y (b) en cuatro puntos característicos de la sección centro luz a lo largo del tiempo. 
a) $2067 \mathrm{~N}$ por metro lineal correspondientes al peso propio de la viga de acero y $12704 \mathrm{~N}$ por metro lineal correspondientes al peso propio de la losa de hormigón.

b) $9838 \mathrm{~N}$ por metro lineal correspondiente al peso del pavimento.

Adicionalmente, se ha considerado una carga de $5000 \mathrm{~N}$ por metro lineal encima de las vigas extremas (vigas 1 y 5 ) correspondiente al peso de la barrera de seguridad situada en los extremos del tablero. No obstante, la consideración de esta carga no tiene consecuencias prácticas por ser las vigas 2 y 3 las más desfavorables por su mayor solicitación térmica.

\subsection{Valoración del fallo}

Se han considerado como criterios de fallo los propuestos por Payá-Zaforteza y Garlock (13). La estructura colapsa cuando se alcanza la deformación última, $\varepsilon_{u}$, del material o se vuelve inestable. Este hecho se manifiesta en forma de un rápido incremento de la flecha máxima registrada en la viga, de un desplazamiento del extremo libre (oeste) hacia el centro de la viga, de un pandeo lateral o de una abolladura del alma. En ocasiones se combinan varios de estos fenómenos.

\subsection{Análisis estructural}

Este artículo analiza la respuesta del puente de la Figura 2 en siete escenarios de fuego, seis de ellos son reales (fire1 a fire6) y otro es una curva de fuego de la normativa -curva de hidrocarburos del EC-1 (3)- empleada por Payá-Zaforteza y Garlock (13) como primera aproximación al problema aunque no está pensada para su aplicación en puentes. Además, se estudian dos posibles condiciones de contorno en lo que respecta a la dilatación del tablero (libre o coaccionada) y cinco niveles de tramificación de la carga térmica.

La Tabla 1 contiene una relación de los casos analizados con los parámetros que los definen y con los principales resultados obtenidos. En ella se incluye el tiempo que tarda la viga analizada en contactar con el estribo adyacente $\left(t_{c}\right)$, el tiempo de fallo $\left(t_{f}\right)$, el desplazamiento longitudinal máximo alcanzado $\left(u_{x}\right)$, la flecha máxima en el momento de fallo $\left(\mathrm{f}_{\max }\right) \mathrm{y}$ los modos de fallo. En la Figura7 se muestran ejemplos de los distintos modos de fallo observados: (a) plastificaciones en las proximidades del apoyo oeste, (b) plastificaciones en centro-luz, (c) plastificaciones en el apoyo este (d) abolladura y (e) pandeo lateral. Los resultados obtenidos se comentan a continuación.

Tabla 1. Casos analizados y resultados.

\begin{tabular}{|c|c|c|c|c|c|c|c|c|c|}
\hline Nombre Análisis & $\begin{array}{l}\text { Escenario } \\
\text { de fuego }\end{array}$ & Tramos & Dilatación & Viga & $t_{c}(\min )$ & $t_{F}(\min )$ & $\mathbf{f}_{\text {MAX }}(\mathbf{m})$ & $\mathbf{u}_{\mathrm{x}}(\mathrm{m})$ & Fallo $^{1}$ \\
\hline hidroc-1-fix-vo & hidroc & 1 & coaccionada & $\mathrm{O}^{2}$ & 1,87 & 4,11 & $-0,058$ & $-0,036$ & $\mathrm{E}$ \\
\hline hidroc-1-free-vo & hidroc & 1 & libre & $\mathrm{O}^{2}$ & - & 8,08 & $-1,195$ & $-0,222$ & CL \\
\hline fire1-16-fix-v3 & fire1 & 16 & coaccionada & 3 & 0,88 & 3,66 & $-0,074$ & $-0,036$ & E, PL \\
\hline fire1-16-free-v3 & fire1 & 16 & libre & 3 & - & 3,20 & $-0,576$ & $-0,188$ & CL \\
\hline fire2-16-fix-v3 & fire2 & 16 & coaccionada & 3 & 0,88 & 2,73 & $-0,177$ & $-0,036$ & $\mathrm{O}, \mathrm{A}, \mathrm{PL}$ \\
\hline fire2-16-free-v3 & fire2 & 16 & libre & 3 & - & 2,84 & $-0,222$ & $-0,116$ & $\mathrm{O}$ \\
\hline fire $3-16-f i x-v 3$ & fire3 & 16 & coaccionada & 3 & 1,00 & 3,19 & $-0,032$ & $-0,036$ & $\mathrm{E}, \mathrm{PL}$ \\
\hline fire3-16-free-v3 & fire3 & 16 & libre & 3 & - & 3,49 & $-0,592$ & $-0,186$ & CL \\
\hline fire4-16-fix-v2 & fire4 & 16 & coaccionada & 2 & 1,04 & 2,79 & $-0,047$ & $-0,036$ & E,PL \\
\hline fire4-16-free-v2 & fire4 & 16 & libre & 2 & - & 3,84 & $-0,596$ & $-0,191$ & CL \\
\hline fire $5-16-f i x-v 2$ & fire 5 & 16 & coaccionada & 2 & 0,92 & 3,00 & $-0,178$ & $-0,036$ & $\mathrm{O}, \mathrm{A}, \mathrm{PL}$ \\
\hline fire5-16-free-v2 & fire5 & 16 & libre & 2 & - & 3,12 & $-0,221$ & $-0,117$ & $\mathrm{O}$ \\
\hline fire6-16-fix-v2 & fire6 & 16 & coaccionada & 2 & 1,23 & 6,89 & $-0,050$ & $-0,036$ & PL \\
\hline fire6-16-free-v2 & fire6 & 16 & libre & 2 & - & 5,55 & $-0,736$ & $-0,209$ & CL \\
\hline fire $1-8-$ fix-v3 & fire1 & 8 & coaccionada & 3 & 0,88 & 3,884 & $-0,099$ & $-0,036$ & E, PL \\
\hline fire $1-4-$ fix-v3 & fire1 & 4 & coaccionada & 3 & 0,88 & 3,915 & $-0,132$ & $-0,036$ & $\mathrm{E}, \mathrm{PL}$ \\
\hline fire $1-2-$ fix-v3 & fire1 & 2 & coaccionada & 3 & 0,88 & 2,385 & $-0,067$ & $-0,036$ & $\mathrm{E}, \mathrm{PL}$ \\
\hline fire1-1-fix-v3 & fire1 & 1 & coaccionada & 3 & 0,89 & 2,506 & $-0,070$ & $-0,036$ & $\mathrm{E}, \mathrm{PL}$ \\
\hline fire2-8-fix-v3 & fire2 & 8 & coaccionada & 3 & 0,89 & 2,775 & $-0,184$ & $-0,036$ & $\mathrm{O}, \mathrm{A}, \mathrm{PL}$ \\
\hline fire $2-4-$ fix $-v 3$ & fire2 & 4 & coaccionada & 3 & 0,88 & 2,945 & $-0,164$ & $-0,036$ & $\mathrm{O}, \mathrm{PL}$ \\
\hline fire $2-2-$ fix $-v 3$ & fire2 & 2 & coaccionada & 3 & 0,88 & 3,140 & $-0,151$ & $-0,036$ & $\mathrm{E}, \mathrm{PL}$ \\
\hline fire2-1-fix-v3 & fire2 & 1 & coaccionada & 3 & 0,87 & 2,228 & $-0,064$ & $-0,036$ & $\mathrm{E}$ \\
\hline
\end{tabular}

${ }^{1}$ PL: pandeo lateral, A: abolladura, E: plastificaciones en el apoyo este, CL: plastificaciones en centro luz, O: plastificaciones en el apoyo oeste.

${ }^{2}$ vo: viga tipo con las cargas gravitatorias de la viga 2 y sometida a la curva de hidrocarburos del EC1 (4). 

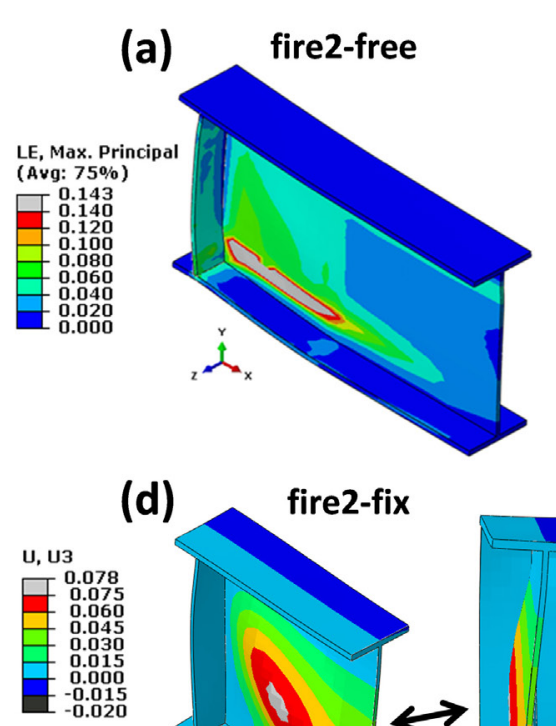

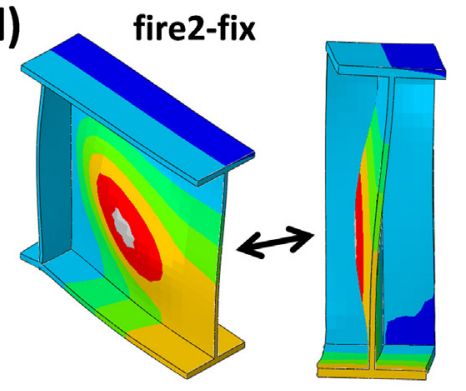

(b) fire1-free

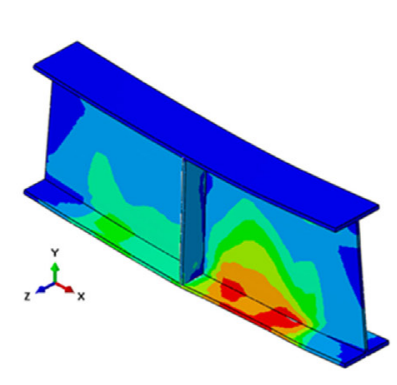

(e) (c) fire3-fix

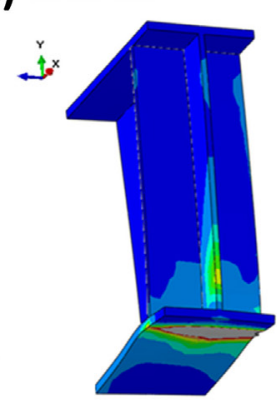

fire1-fix

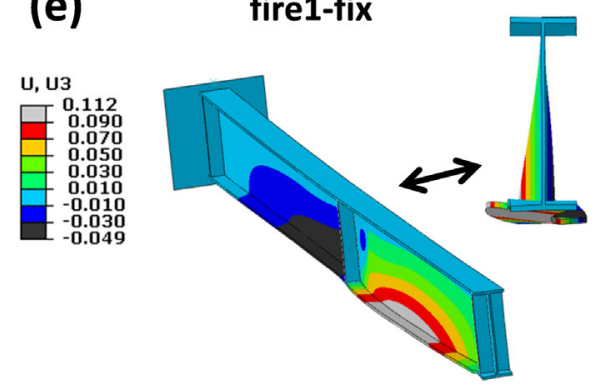

Figura 7. Tipos de fallo detectados: (a) plastificaciones en las proximidades del apoyo oeste, (b) plastificaciones en centro-luz, (c) plastificaciones en el apoyo este (d) abolladura y (e) pandeo lateral.

\subsubsection{Influencia de la tramificación de las curvas}

En el programa Abaqus no se pueden introducir las curvas de fuego correspondientes a escenarios reales obtenidas con FDS de forma directa. Por ello, es necesario discretizar la viga en una serie de tramos en cada uno de los cuales se asume una carga de fuego uniforme. Esta carga se corresponde con la curva de temperaturas medias en el tramo. Por este motivo se ha hecho un análisis para ver la importancia de la tramificación en los resultados. Se han propuesto discretizaciones de 1, 2, 4, 8 y 16 tramos correspondientes a anchos de tramo de $0,76,1,52,3,05$, 6,10 y $12,20 \mathrm{~m}$. De esta manera se analiza si pueden emplearse discretizaciones groseras que reduzcan tiempos de modelado y de cálculo. En la Figura 8 se puede ver cómo han sido adaptadas las curvas obtenidas mediante FDS a las restricciones de caracterización de las condiciones de contorno térmicas en el programa Abaqus. El estudio se ha realizado con los fuegos fire1 y fire2 y considerando únicamente modelos de dilatación coaccionada.

La Figura 9 muestra la evolución de las flechas máximas en la viga analizada a lo largo del incendio para los dos escenarios considerados y los modelos tipo coaccionado. La figura permite comprobar que la respuesta estructural en los escenarios de fuego fire1 y fire2 es diferente. Mientras que en el incendio provocado por el camión centrado (fire1) las flechas suelen ser superiores con tramificaciones más groseras, en el incendio asociado al camión en el extremo oeste (fire2) las flechas se van reduciendo conforme se reduce el número de tramos con la excepción del caso de tramo único. Adicionalmente se ha detectado que una tramificación de 8 subdivisiones presenta resultados muy similares a la tramificación de 16 tramos en cualquiera de los dos incendios por lo que podría considerarse la reducción del número de tramos necesarios para estos dos escenarios de fuego. Las tramificaciones en 1 y 2 subdivisiones quedarían totalmente descartadas por imprecisas, mientras que la tramificación en 4 tramos no acaba de reproducir exactamente el fenómeno que se produce antes del colapso en el incendio fire1.

En el presente estudio, salvo los modelos que consideran el escenario de hidrocarburos y los que indican expresamente el número de tramos, los análisis han incorporado una discretización en 16 subdivisiones con el fin de reproducir lo más fielmente posible la respuesta estructural.

\subsubsection{Influencia del escenario de fuego y de la coacción a la dilatación del tablero}

Se analiza seguidamente la influencia en la respuesta del tablero de la posición de la carga de fuego y del tipo de restric- (a)

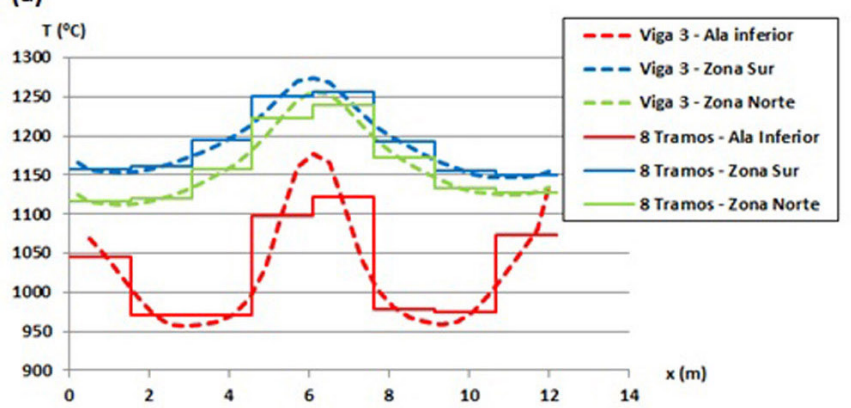

(b)

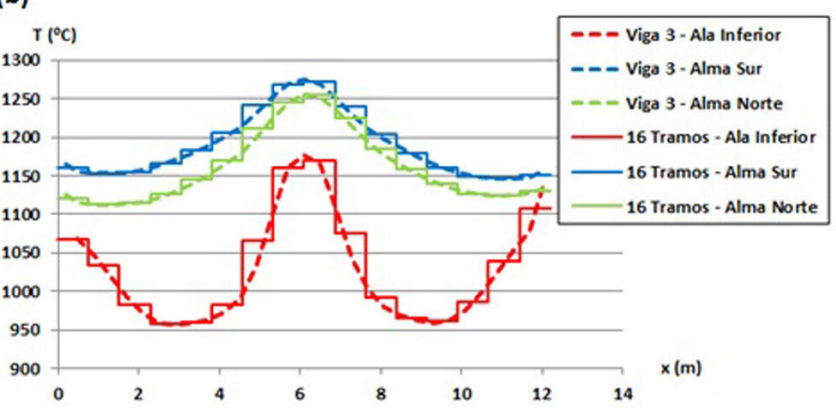

Figura 8. Ejemplo de tramificación de carga térmica para el escenario fire1: (a) 8 tramos de 1,52 m. (b) 16 tramos de o,76 m. 
(a)

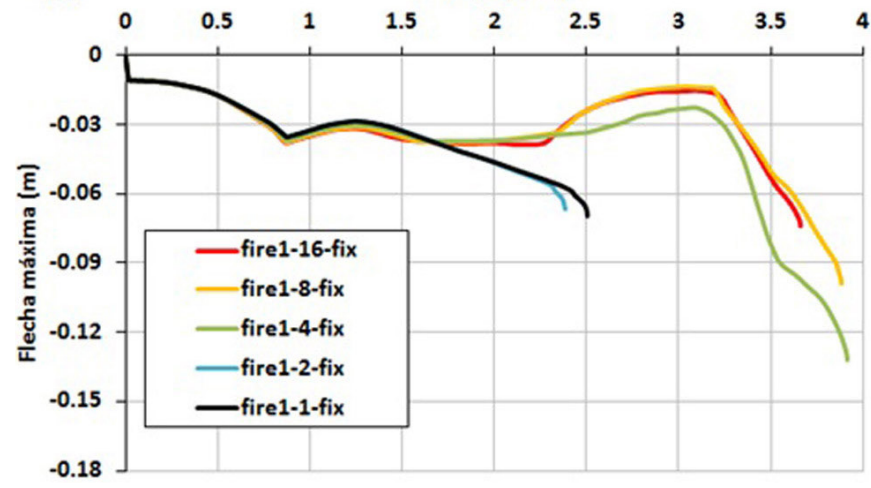

(b) tiempo (min)

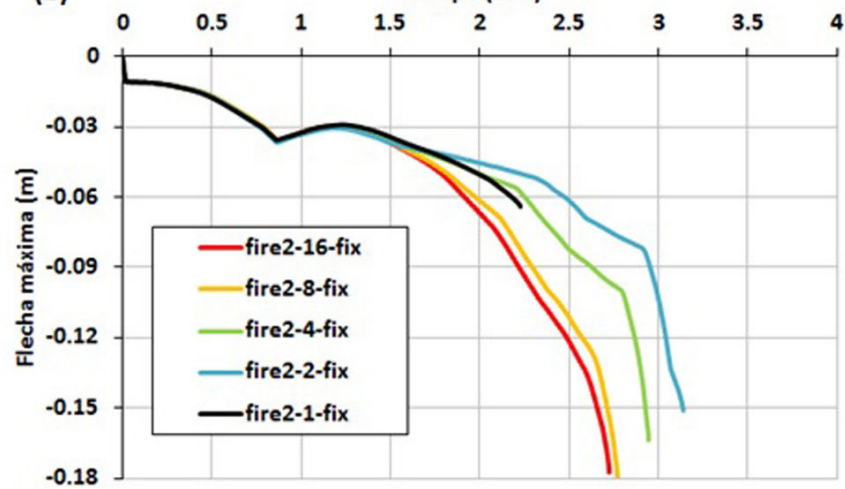

Figura 9. Evolución flecha máxima de la viga 3 con distintas tramificaciones para los casos: (a) fire1 (b) fire2.

ción longitudinal considerada en el extremo del puente donde se ubica el apoyo móvil. Se han estudiado siete casos diferentes de carga de fuego, seis corresponden a fuegos reales y son los indicados en la Figura 3. El séptimo caso se corresponde con la curva de hidrocarburos propuesta por el Eurocódigo 1 (4). Para todos los casos de fuego se ha estudiado el efecto de la coacción longitudinal a la dilatación longitudinal del tablero teniendo en cuenta los dos opciones explicadas en el apartado 4.2: dilatación libre (casos free) y dilatación máxima fijada por el ancho de la junta de dilatación (casos fix).

La Figura 10 muestra la evolución en el tiempo de los desplazamientos horizontales y verticales máximos en los escenarios de fuego analizados para las dos posibles hipótesis de coacción de la dilatación del tablero (libre y coaccionada).
Para los casos fire1 a fire3 las gráficas muestran la respuesta estructural de la viga 3 mientras que para los casos fire 4 a fire6 la respuesta estructural corresponde a la viga 2. En ambos casos se ha presentado la respuesta de la viga sometida a la mayor carga térmica. Se detallan seguidamente las principales conclusiones que se pueden extraer de los resultados obtenidos.

Para los casos sin coacción longitudinal, el tiempo de fallo oscila entre 2,8 y 5,5 minutos para las hipótesis de incendios reales, mientras que la curva de hidrocarburos presenta una resistencia a fuego de 7,8 minutos. Resulta interesante indicar que la respuesta que presenta la viga de estudio sometida a una curva de hidrocarburos ofrece un tiempo de fallo notablemente superior (entre un 41 y un $180 \%$ ) al obtenido para las seis

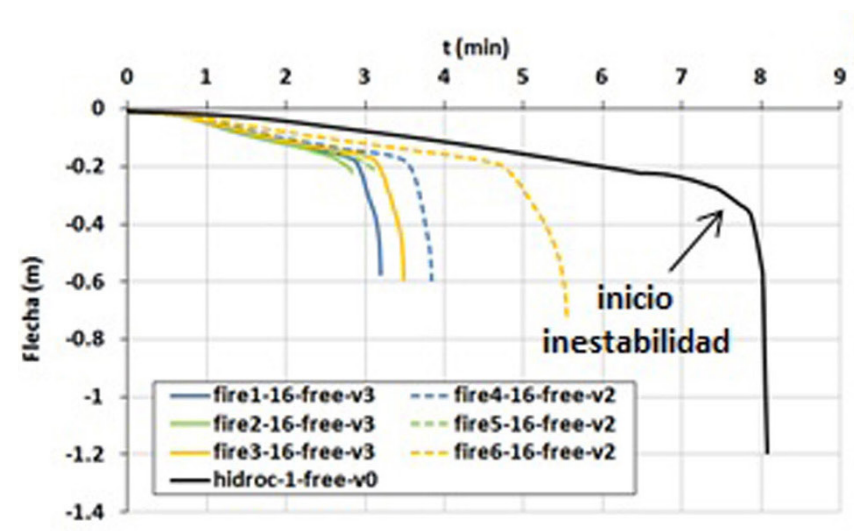

LIBRE

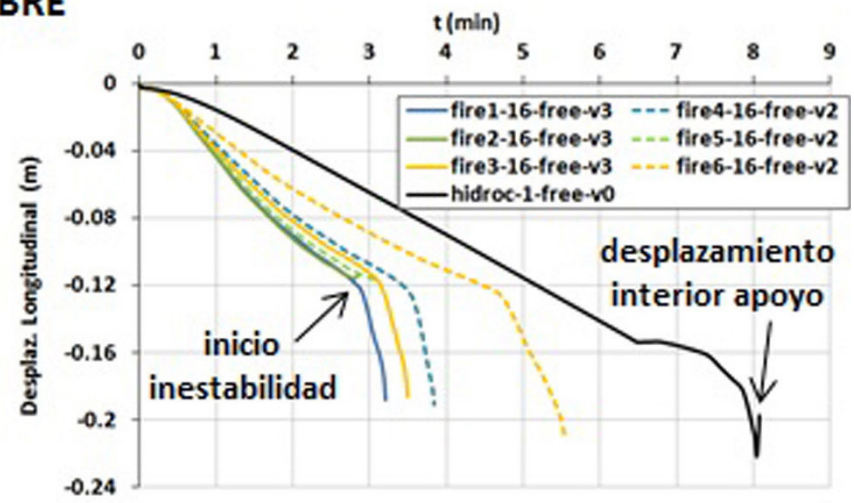

COACCIONADA
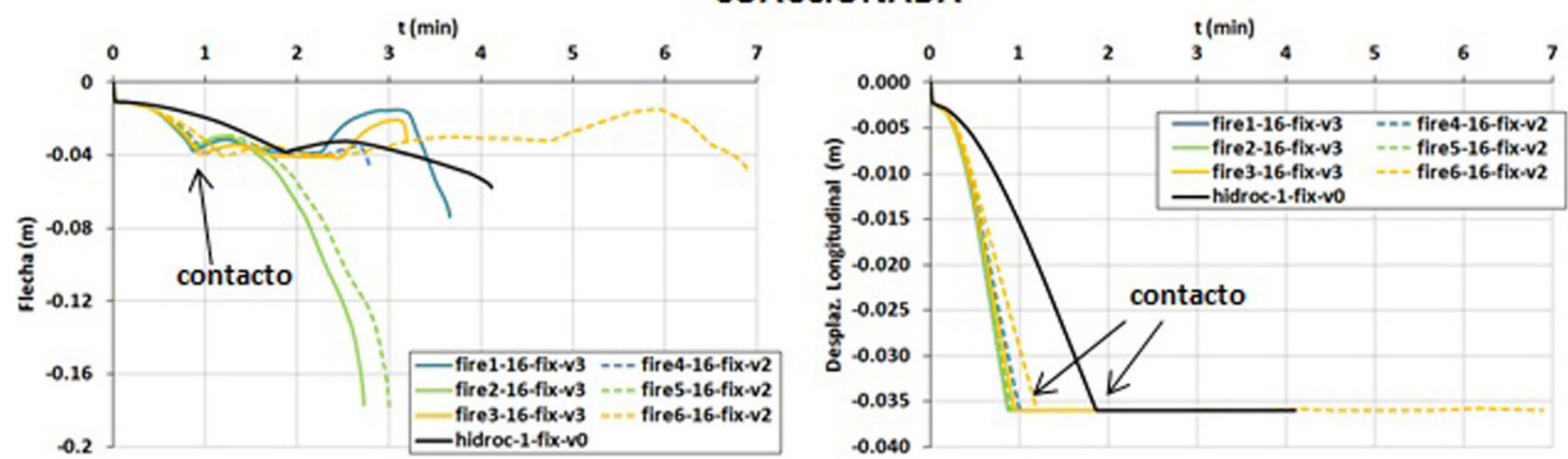

Figura 10. Evolución de las flechas máximas, parte izquierda (a) y (c), y de los desplazamientos longitudinales, parte derecha (b) y (d), para las condiciones de contorno y escenarios de fuego considerados. En la parte superior se muestra el caso de dilatación libre - casos free (a) y (b) - y en la parte inferior el caso de dilatación coaccionada -casos fix, (c) y (d)-. 
hipótesis de incendio consideradas. Se observa que los desplazamientos máximos longitudinales detectados para la condición de dilatación libre para las siete hipótesis de incendio son notablemente superiores al tamaño de la junta de dilatación.

Para los casos con coacción longitudinal fire1 a fire 5 los tiempos de fallo oscilan entre 2,7 y 3,6 minutos, mientras que en el escenario fire6 la resistencia el fuego se eleva hasta $6,9 \mathrm{mi}-$ nutos como consecuencia del calentamiento más progresivo al que se somete a la viga con respecto al resto de casos (Figura 5). En cuanto al escenario de hidrocarburos presenta un tiempo de fallo de 4,1 minutos, menor que en el caso libre y mayor que los casos fire1 a fire (entre un 14 y un 52 \%) apreciándose además que estos porcentajes son inferiores a los registrados en los casos libres. Los mayores tiempos de fallo para el caso de hidrocarburos se deben fundamentalmente a que (a) la curva de hidrocarburos es una acción térmica uniforme a lo largo de la viga, menos perjudicial que una acción variable como la que se produce en los incendios reales y (b) la curva de hidrocarburos presenta un calentamiento más progresivo.

El tiempo transcurrido hasta que el extremo oeste de la viga contacta con la junta de dilatación oscila entre 0,9 y 1,2 minutos para los escenarios de fuego reales mientras que en la hipótesis de hidrocarburos se sitúa en 1,9 minutos (entre un 58 \% y $111 \%$ superior). El mayor tiempo de contacto requerido en la hipótesis de hidrocarburos se debe únicamente a que la curva de calentamiento es más progresiva que las obtenidas en los escenarios de fuego reales. En la Figura 11a puede verse como las curvas de calentamiento aplicadas sobre la viga en el escenario fire1 alcanzan el máximo muy rápidamente como consecuencia de la gran velocidad de propagación $(\sim 2$ $\mathrm{m} / \mathrm{s}$ ) (20) de la llama en combustibles como la gasolina. Este hecho hace que el incendio adquiera rápidamente el carácter de totalmente desarrollado con las llamas muy próximas a la viga de estudio. Resulta necesario por tanto, el desarrollo de curvas de fuego específicas para puentes con el fin de evaluar su resistencia.

En lo que respecta a la posición transversal de la carga de fuego, para el caso de dilatación libre, se detecta que los tiempos de fallo son menores cuando la carga se encuentra próxima a los estribos y mayores para el caso de carga centrada en el vano quedando el caso con derrame en una situación intermedia. Se ha detectado, además, cómo la plastificación suele producirse en la región de centro luz ocasionando un aumento de flecha considerable, con la excepción de los escenarios fire2 y fire5 en que las cargas de fuego se sitúan próximas a los estribos haciendo fallar a la pieza por el extremo más solicitado térmicamente. La causa es un mayor deterioro del material como consecuencia de encontrarse sometido a mayores temperaturas, alcanzando el fallo con flechas menores.

En los casos con dilatación del tablero coaccionada, la posición de la carga afecta al tipo de fallo. Para los casos con camión cisterna en centro-luz el fallo se produce por plastificación del apoyo este, debido a la alta concentración de tensiones que aparecen. En el caso de camión cisterna cerca del estribo, se producen fenómenos de abolladura combinados con plastificaciones en la zona del apoyo oeste.

Cabe destacar que los casos con derrame (fire3 y fire6) no son más desfavorables que sus respectivos casos de carga centrada (fire1 y fire4) como cabría esperar dada la mayor tasa de liberación de calor de estos casos. La causa reside en que al incorporar una nueva superficie emisora de calor se modifican los flujos existentes bajo el puente de forma que se producen velocidades mayores (Figura 11b) que dificultan la acumulación de calor con respecto a los casos sin derrame con carga centrada. Los tiempos de fallo de la viga más desfavorable son menores cuando el penacho se sitúa centrado en la viga 3.

En los casos coaccionados, el desplazamiento longitudinal se ve restringido a $0,036 \mathrm{~m}$. desde el instante en que la viga contacta con el estribo. Debido a esto, y viendo que en los casos libres la viga tiende a desplazamientos en el entorno de 0,20 $\mathrm{m}$, se introducen unas fuertes compresiones que son soportadas por el apoyo este. A consecuencia de éstas se produce el fenómeno de pandeo lateral en todos los escenarios de fuego real. En el caso de hidrocarburos no se produce este fenómeno porque el pandeo lateral requiere además de la existencia de una diferencia de temperaturas entre las dos regiones.

\section{CONCLUSIONES}

Este artículo analiza la respuesta al fuego de un puente multijácena metálico simplemente apoyado de 12,2 m de luz sometido a distintos escenarios de incendio incluyendo casos con y sin derrame de combustible en distintos emplazamientos. Los modelos de fuego emplean técnicas de dinámica de fluidos computacional implementadas en el programa FDS y la respuesta termo-estructural se analiza con modelos de elementos finitos implementados en Abaqus. (a)

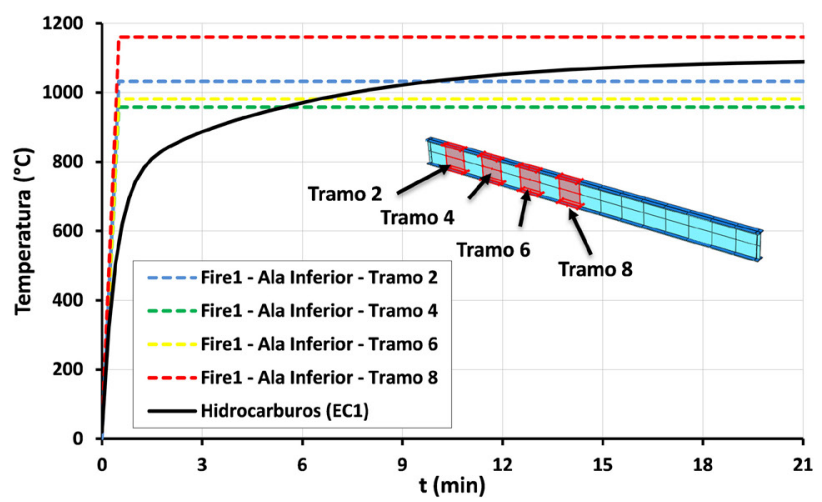

(b)
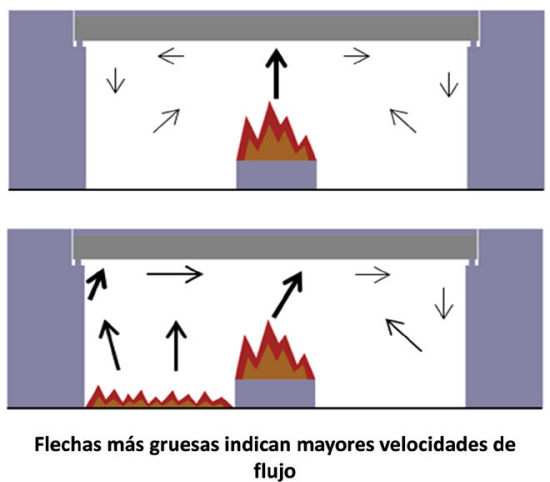

Figura 11. (a) Comparación de las curvas de calentamiento de los tramos 2, 4, 6 y 8 obtenidas en el escenario fire1 con la curva de hidrocarburos. (b) Croquis de las velocidades de flujo en un incendio con y sin derrame de combustible. 
Los resultados obtenidos permiten extraer las conclusiones siguientes:

1) Los escenarios de fuego reales, tanto en condiciones de dilatación libre como dilatación restringida, presentan tiempos de fallo inferiores a los que ofrecería un análisis adoptando la curva de hidrocarburos siendo la reducción de tiempos de fallo mucho más notable en los casos de dilatación libre. Esto se debe a que la curva hidrocarburos tiene un incremento de temperaturas más progresivo. Es necesario, por tanto, la inclusión en las normativas de curvas de fuego que se adapten mejor a los escenarios reales de fuego en puentes.

2) En los modelos con dilatación libre el colapso se produce por plastificación en la región de centro del vano en los casos de carga centrada con y sin derrame mientras que en los casos de carga próxima al estribo oeste las plastificaciones se producen en las proximidades del apoyo oeste.

3) En los modelos con dilatación coaccionada el colapso se produce en el extremo este en la zona donde se materializa el apoyo fijo por concentración de tensiones, con la excepción de los casos con carga próxima al estribo oeste donde el fallo se produce por plastificación y abolladura en las proximidades del apoyo oeste.

4) Una restricción longitudinal al movimiento, siempre que no implique una compresión excesiva de la pieza que genere tensiones excesivas en el apoyo fijo, resultará positiva tanto en lo que respecta a tiempos de fallo como en lo referente a flechas máximas previas al fallo. En cambio, si la compresión introducida es muy elevada provocará la movilización de los mecanismos de abolladura y pandeo lateral. En el caso particular del pandeo lateral se ha detectado como los rigidizadores situados en centro luz influyen en el modo de pandeo.

5) La posición de la carga de fuego y la existencia del derrame son variables esenciales tanto en la caracterización como en la determinación de la viga más solicitada térmicamente. Una tasa de liberación de calor total superior no necesariamente implicará temperaturas superiores puesto que variables como la velocidad y la oposición total o parcial al flujo son parámetros también fundamentales.

6) La implementación de las curvas obtenidas mediante FDS en el programa Abaqus no es directa, por lo que debe escogerse una discretización en tramos suficiente fina para garantizar una respuesta estructural acorde con la realidad. En el presente estudio se ha visto que una división en tramos de 1,52 metros (equivalentes a $\mathrm{L} / 8$ donde $\mathrm{L}$ es la luz del puente) en un puente de un solo vano de 12,2 metros de luz con una carga de fuego promedio de $72 \mathrm{MW}$, proporciona resultados suficientemente precisos.

7) Aunque el presente artículo muestra la posibilidad de simular incendios reales en puentes, resulta evidente la necesidad de incorporar en la normativa herramientas y modelos simplificados que permitan un diseño basado en prestaciones de los puentes con un enfoque similar al empleado en la edificación.

Las conclusiones anteriores se consideran válidas para puentes de geometría y dimensiones similares a las del analizado, pero son necesarios estudios adicionales para extenderlas a puentes de luces mayores o con características particulares.

\section{AGRADECIMIENTOS}

Este trabajo ha sido posible gracias a la financiación prestada por el Ministerio de Ciencia e Innovación (proyecto BIA 2011-27104 del Plan Nacional de I+D+i) y por la Universitat Politècnica de València (Programa de Apoyo a la I+D PAID06-11). Los autores desaean agradecer la colaboración en los modelos de fuego prestada por D. Eduardo Loma de Osorio, ingeniero del Cuerpo de Bomberos de Valencia.

\section{REFERENCIAS}

(1) Ministerio de Fomento. (2011). Instrucción sobre las acciones a considerar en el proyecto de puentes de carretera IAP-11. Madrid: Ministerio de Fomento.

(2) CEN. (2003). Eurocode 1 Actions on Structures, Part 2: Traffic loads on bridges. Bruselas (Bélgica): European Committee for Standardization (CEN).

(3) Priestley, M.J.N., Seible, F., Calvi, G.M. (1996). Seismic design and retrofit of bridges. New York (USA): John Wiley \& Sons Inc.

(4) CEN. (2002). Eurocode 1 Actions on Structures, Part 1-2: General Actions - Actions on Structures Exposed to Fire. Bruselas (Bélgica): European Committee for Standardization (CEN).

(5) Orta, B. (2010). El tiempo equivalente de exposición al fuego en un edificio histórico. Informes de la Construcción, 62(520): 57-66, doi: http://dx.doi.org/10.3989/ic.09.060.

(6) Quiel, S.E., Garlock, M.E., Paya-Zaforteza I. (2011). Closed form procedure for predicting the capacity and demand of steel beam-columns under fire. ASCE Journal of Structural Engineering, 137(9): 967-976, doi: http://dx.doi.org/10.1061/ (ASCE)ST.1943-541X.0000443.

(7) Espinós, A., Romero, M. L., Hospitaler, A. (2012). Simple calculation model for evaluating the fire resistance of unreinforced concrete filled tubular columns. Engineering Structures, 42: 231-244, doi: http://dx.doi.org/10.1016/j.engstruct.2012.04.022.

(8) López-Colina C., Serrano-López M.A., Gayarre F.L., del Coz-Díaz J.J. (2011). Stiffness of the component 'lateral faces of RHS' at high temperature. Journal of Constructional Steel Research, 67(12): 1835-1842, doi: http://dx.doi. org/10.1016/j.jcsr.2011.05.006.

(9) Garlock, M.E, Paya-Zaforteza, I., Kodur, V., Gu, L. (2012). Fire hazard in bridges: Review, assesment and repair strategies. Engineering Structures, 35: 89-98, doi: http://dx.doi.org/10.1016/j.engstruct.2011.11.002. 
(10) New York State Department of Transportation. (2011). Bridge fire incidents in New York State (Private Correspondence with Prof. M. Garlock). USA: New York State Department of Transportation.

(11) ABC Action News (2012). Driver killed in fiery truck crash on NJ Tumpike. http://6abc.com/archive/8833938/.

(12) Paya-Zaforteza, I., Alós-Moya, J., Hospitaler, A. (2012). Puentes y Fuego. Análisis de una problemática. En II Fïre Engineering Conference, Valencia.

(13) Paya-Zaforteza, I., Garlock, M.E. (2012) A numerical investigation on the fire response of a steel girder bridge. Journal of Constructional Steel Research, 75: 93-103, doi: http://dx.doi.org/10.1016/j.jcsr.2012.03.012.

(14) AISC. (2010). Steel Construction Manual (14th Edition). Chicago, IL - USA: American Institute of Steel Construction.

(15) ASTM. (2004). ASTM A6/A6M Standard Specification for Structural Steel (A36-/A36M-o4a). West Conshohocken, PA - USA: American Society for Testing and Materials (ASTM).

(16) NIST. (2007). Fire Dynamics Simulator (version 5) User's Guide. National Institute of Standards and Technology. http://fire.nist.gov/bfrlpubs/fireo7/PDF/f07053.pdf.

(17) Simulia. (2011). Abaqus 6.11: Abaqus/CAE User's Manual. http://abaqus.ethz.ch:208o/v6.11/pdf_books/CAE.pdf.

(18) Alos-Moya, J., Paya-Zaforteza I., Garlock M.E.M., Loma-Ossorio E., Schiffner D., Hospitaler A. (2014). Analysis of a bridge failure due to fire using computational fluid dynamics and finite element models. Engineering Structures, 68: 96-110, doi: http://dx.doi.org/10.1016/j.engstruct.2014.02.022.

(19) Catalogo hytruck, modelo, Euro II Hongyan XinDaKang. http://www.hytruck.es.

(20) SFPE. (2002). SFPE Handbook of Fire Protection Engineering (3th edition). Society of Fire Protection Engineers.

(21) CEN. (2011). Eurocódigo 2: Proyecto de estructuras de hormigón, Parte 1.2: Reglas Generales, Proyecto de estructuras sometidas al fuego. Bruselas (Bélgica): European Committee for Standardization (CEN).

(22) CEN. (2011). Eurocódigo 3 Proyecto de estructuras de acero Parte 1.2: Reglas generales, Proyecto de estructuras sometidas al fuego. Bruselas (Bélgica): European Committee for Standardization (CEN).

(23) Peris-Sayol, G., Alós-Moya, J., Paya-Zaforteza, I., Hospitaler, A. (2013). Análisis numérico de la respuesta térmica y estructural de un puente de vigas metálico en condiciones reales de incendio. En II International Congress on Mechanical models in structural engineering.

(24) Wickström, U. (2007). Adiabatic surface temperature for calculating heat transfer to fire exposed structures. Interflam, 2: $943-953$ 